\title{
Designing Enzyme-Like Catalysts: A Rhodium(II) Metallopeptide Case Study
}

\author{
Farrukh Vohidov', Brian V. Popp ${ }^{2}$, and Zachary T. Ball ${ }^{*}$ \\ ${ }^{1}$ Rice University, Department of Chemistry, Houston, TX, 77005, USA; ${ }^{2}$ West Virginia University, \\ C. Eugene Bennett Department of Chemistry, Morgantown, WV, 26506, USA
}

\section{Introduction}

Peptides are relatively simple molecules with remarkable capacity for supramolecular assembly and molecular recognition. In this study, harnessing the combined potential of peptide molecular recognition and unique coordination chemistry or catalysis with non-biological transition metals allows the development of new tools for protein chemistry and probe development. While methods for chemical manipulation of recombinant proteins are well established [4,5], natural proteins, which are without engineered sequences or side-chain handles, remain a challenging target for chemical manipulation.

\section{Results and Discussion}

We began efforts at selective protein modification with transition-metal catalysts by focusing on rhodium(II) metallopeptides. There was established reactivity of these complexes toward tryptophan side chains [6], and we developed robust, general, and reliable methods for the synthesis and purification of rhodium(II) metallopeptides [7-9]. The metalation of unprotected peptides did not affect folding, and could even be used to enforce helical structure [7].

In orienting experiements, we focused on the modification of helical peptides that self-assemble into heterodimeric coiled coils in the presence of complementary metallopeptides (Figure 1). Treatment with stabilized diazo reagents resulted in clean modification of position "g" on the corresponding substrate helix, immediately flanking the rhodium center. The site of location was established by MS/MS fragmentation methods [1,2] and NMR with isotopically-labeled diazo reagents. Tryptophan was the most reactive side chain. Turnover numbers of up to 50 were obtained at micromolar substrate concentration, and the rate enhancement for complementary substrates relative to an unstructured control reaction was $>10^{3}$. Even more powerfully, the proximity-driven catalysis enabled modification of many other side chains (Figure 1) that are completely unreactive with simple

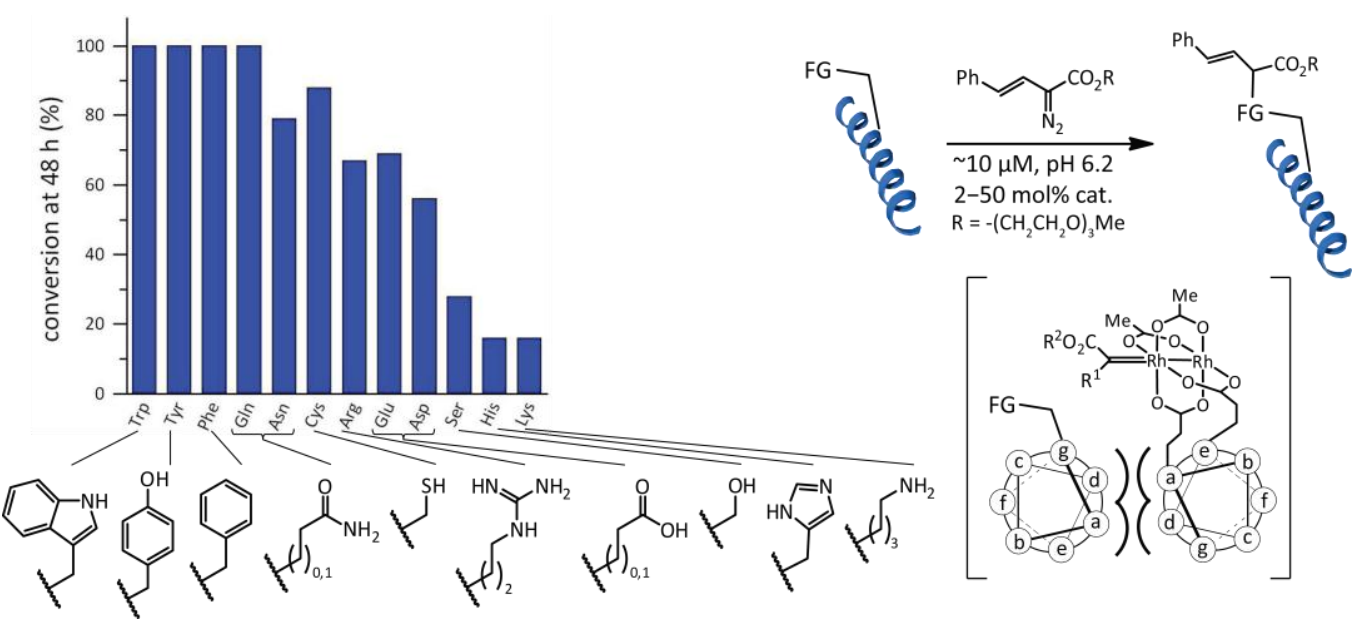

Fig. 1. Site-specific covalent modification of helical peptides catalyzed by rhodium(II) metallopeptides enables remarkably broad and selective amino-acid reactivity. "Conversion" indicates reaction at position " $g$ " of a helix. Site of modification is established by MS/MS fragmentation analysis [1,2]. 
rhodium catalysts such as $\mathrm{Rh}_{2}(\mathrm{OAc})_{4}$. In totality, residues identified as substrates for modification make up $>40 \%$ of protein amino-acid space. The reactivity demonstrated here enabled a number of studies with similar engineered coils, including design of orthogonally reactive catalyst-substrate [10] pairs and the use of tryptophan-containing coils as tags for proteins expressed in lysate $[10,11]$.

Importantly, these initial studies could be successfully extended from engineered coils to the modification of completely natural protein substrates by employing protein-peptide recognition motifs beyond the coiled coil. We examined modification of SH3 domains in significant detail [3]. The SH3 domain recognized short proline-rich motifs in an extended, PPII-helix conformation. Through proper design of catalyst, it is possible to achieve selective reaction at individual members of members of the Src-family kinase SH3 domains. Even more remarkable, we designed a suites of catalysts that selectively modify different individual amino-acids on the Lck SH3 domain, demonstrating that catalyst control is capable of building ensembles of modified proteins, decorated at different sites near the peptide-binding cleft (Figure 2).

The sensitivity and specificity of the modification methods could be probed by examining human cell lystates from cell lines known to express an individual Src-family kinase. As shown (Figure 3) covalent modification of the Yes kinase was visualized by blotting with a fluorogenic dye specific for the chemical modification. The method identifies Yes, which could also be visualized by a traditional antibody blot technique. The experiment demonstrates that chemical modification succeeds on whole human proteins at natural abundance levels. Because the SH3 domain of a full-length kinase is tied up in intramolecular interactions with a kinase domain loop (see structure, Figure 3), these cell lysate results indicate that catalysis is possible even when the metallopeptide and substrate concentrations lie below the dissociation constant for assembly.

Metallopeptide recognition of SH3 domains is one example of practical rhodium(II) catalysts that employ molecular recognition to achieve selectivity. Small-molecule-rhodium conjugates are also effective in cases where small-molecule ligands for protein-protein interaction sites can be found. In addition, SH2 domains, coiled-coil domains, and other well-known peptide-binding motifs represent suitable targets for catalyst development. Current efforts also include the design and discovery of minimalist catalyst recognition motifs on a protein surface.

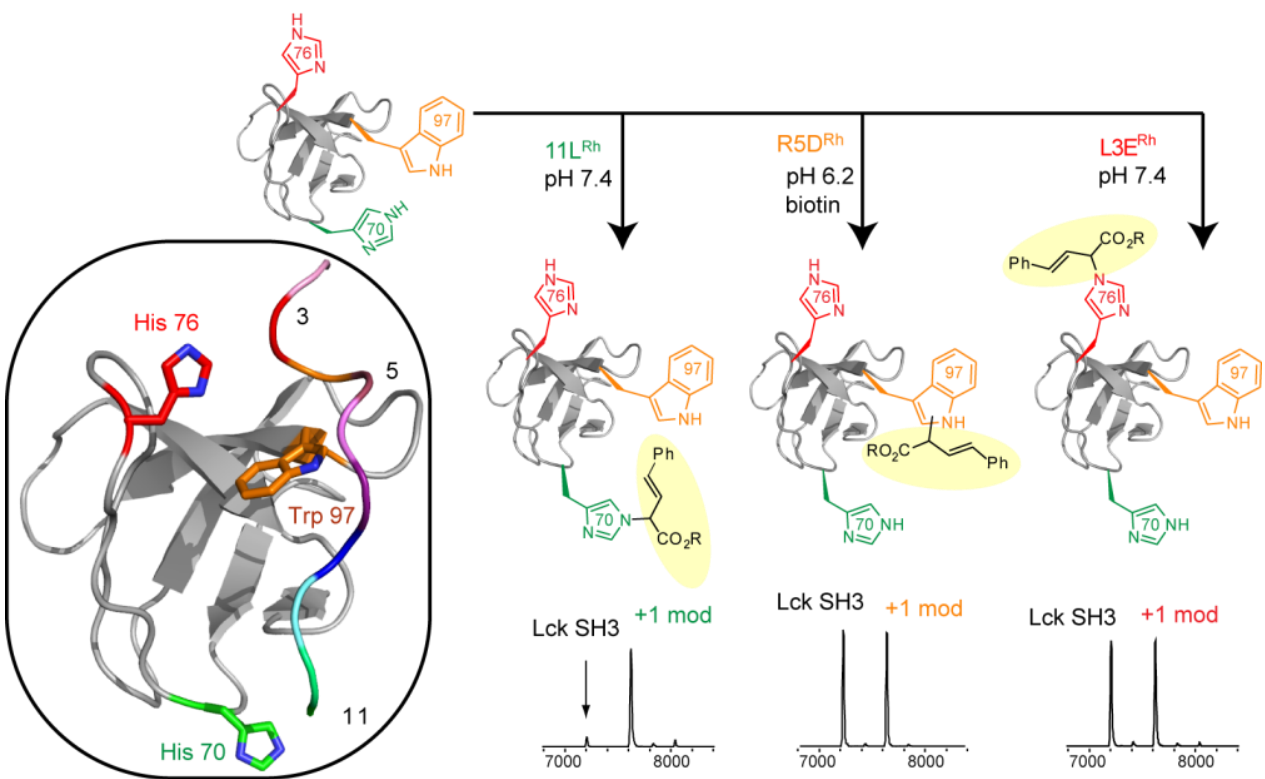

Fig. 2. Site-specific modification different amino-acid sites on the Lck SH3 domain is achieved by catalyst design of rhodium metallopeptides at the indicated amino acids (3, 5, or 11) on a prolinerich SH3-binding peptide. Structure-guided catalyst designed enables independent modification of His70, His76, or Trp97 on the Lck SH3 domain [3]. 


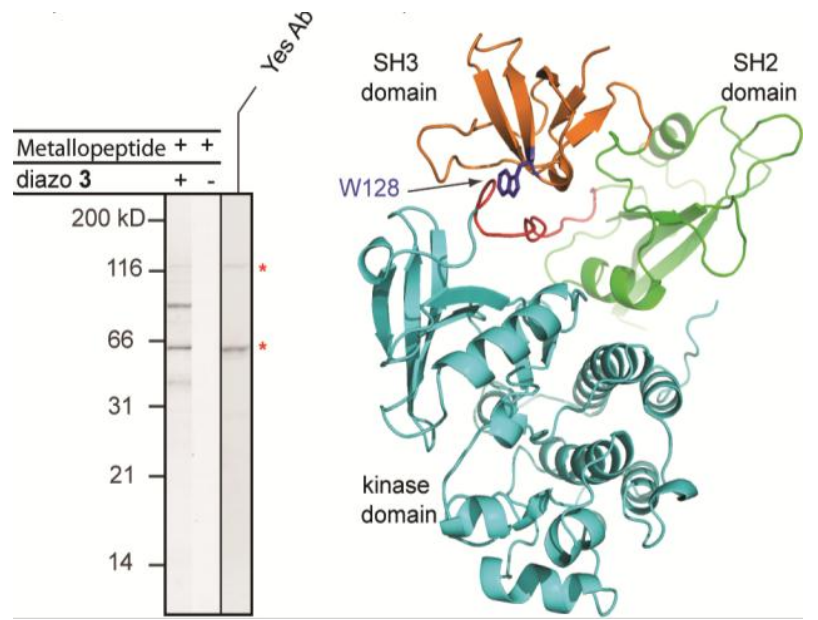

Fig. 3. (left) Gel image demonstrating modification of Yes kinase in human PC-3 cell lysate, with Yes antibody staining at right for comparison. (right) The rest-state structure of Src-family kinases, showing the interaction between the SH3 domain (orange) and a loop region near the kinase domain (cyan), effectively burying the SH3 peptide-binding motif. Shown here for Src (PDB ID: 2SRC).

\section{Acknowledgments}

We acknowledge support from the National Institutes of Health under grant number 5R21CA170625, from the Robert A. Welch Foundation Research Grant C-1680, and from the National Science Foundation (CHE-1055569).

\section{References}

1. Popp, B.V., Ball, Z.T. Chem. Sci. 2, 690-695 (2011), http://dx.doi.org/10.1039/c0sc00564a

2. Popp, B.V., Ball, Z.T. J. Am. Chem. Soc. 132, 6660-6662 (2010), http://dx.doi.org/10.1021/ja101456c

3. Vohidov, F.,Coughlin, J.M., Ball, Z.T. Angew. Chem. Int. Ed. 54, 4587-4591 (2015), http://dx.doi.org/10.1002/anie.201411745

4. Hinner, M.J., Johnsson, K. Curr. Opin. Biotechnol. 21, 766-776 (2010), http://dx.doi.org/10.1016/j.copbio.2010.09.011

5. Sletten, E.M., Bertozzi, C.R. Angew. Chem. Int. Ed. 48, 6974-6998 (2009), http://dx.doi.org/10.1002/anie.200900942

6. Antos, J.M., Francis, M.B. J. Am. Chem. Soc. 126, 10256-10257 (2004), http://dx.doi.org/10.1021/ja047272c

7. Zaykov, A.N.,Popp, B.V., Ball, Z.T. Chem. Eur. J. 16, 6651-6659 (2010), http://dx.doi.org/10.1002/chem.200903092

8. Zaykov, A.N.,MacKenzie, K.R., Ball, Z.T. Chem. Eur. J. 15, 8961-8965 (2009), http://dx.doi.org/10.1002/chem.200901266

9. Zaykov, A.N., Ball, Z.T. Chem. Commun. 47, 10927-10929. http://dx.doi.org/10.1039/c1cc13169a

10. Chen, Z.,Vohidov, F.,Coughlin, J.M., Stagg, L.J., Arold, S.T.,Ladbury, J.E.,Ball, Z.T. J. Am. Chem. Soc.

134, 10138-10145 (2012), http://dx.doi.org/10.1021/ja302284p

11. Chen, Z.,Popp, B.V.,Bovet, C.L.,Ball, Z.T. ACS Chem. Biol. 6, 920-925 (2011), http://dx.doi.org/10.1021/cb2001523 